\title{
Dynamics of Non-Linear Polarisation Rotation in Semiconductor Optical Amplifiers
}

\author{
S. Philippe ${ }^{1 *}$, A.L. Bradley ${ }^{1}$, F. Surre ${ }^{1,2}$, Member, IEEE, B. Kennedy ${ }^{2,3}$, P. Landais ${ }^{2}$, Member, IEEE $^{2}$ \\ ${ }^{1}$ School of Physics, Trinity College Dublin, Dublin, Ireland \\ ${ }^{2)}$ School of Electronic Engineering, Dublin City University, Dublin, Ireland \\ ${ }^{3)}$ Departamento de Ingenieria Electrica, Universidad de Santiago, Chile \\ *Tel: +35316082677,Fax: +35316711759,Email:philipps@tcdie
}

\begin{abstract}
A free space contra-propagation set-up is implemented and a dynamic pump-probe study of a SOA in the picosecond regime is undertaken. The dynamics of the change in the probe state of polarisation is investigated and the implications for polarisation switching discussed. Polarisation resolved detection is shown to enhance switching performance for carefully chosen polarizer orientation.
\end{abstract}

Keywords: semiconductor optical amplifier, gain dynamics, polarisation, pump-probe, optical switching.

\section{INTRODUCTION}

Due to their gain saturation effect, fast response and strong non-linearities, semiconductor optical amplifiers (SOA) are not only suitable for amplification, but also for all-optical signal processing applications. Bit rate capabilities in excess of $100 \mathrm{Gbit} / \mathrm{s}$ have been reported using a variety of schemes [1]. The change of polarisation and the gain experienced by a laser beam as it travels through the SOA are both altered when a second laser beam is injected into the device. This phenomenon, non-linear polarisation rotation, can potentially be used for high speed all-optical switching applications [2-4]. While a large amount of work focussing on its applications has been performed, the underlying physical mechanisms are not thoroughly understood. This is mainly due to the use of optical fibres in most set-ups, which make preserving and tracking the signals' states of polarisation extremely difficult. Therefore a free space configuration is used here allowing for total control and preservation of the state of polarisation of the injected and collected signals

Conventional pump-probe experiments use a co-propagation configuration, where the pump and probe signals travels collinearly through the SOA and are separated at the output using a polarizer. In that case to enable separation of the signals at the output of the SOA it is typical for the pump and probe output states of polarisation to be linear and orthogonal to each other. Consequently the input states of polarisation are restricted to the eigen modes of the device and only two polarization combinations can be studied, pump TE probe TM and pump TM probe TE. No restrictions on the injected signals' polarisation apply when a contra-propagation configuration is used. Using such a configuration, we present a polarisation resolved pump-probe study of a bulk $\mathrm{SOA}$ in the picosecond regime with specific focus on the change of output probe state of polarisation and its dynamics.

\section{EXPERIMENT}

A free space contra-propagation configuration is used, allowing total control and preservation of the state of polarisation of the injected and collected signals, as shown on Fig. $1 a$. The $1580 \mathrm{~nm}$ pulsed input is produced using a fibre based femtosecond laser, with a repetition rate of $82 \mathrm{MHz}$. The pulse width after dispersion in the fiberised output is $2.5 \mathrm{ps}$ and a grating filter with a $1 \mathrm{~nm}$ spectral width is used to select the wavelength. The pulses are coherent and one is delayed with respect to the other using a variable delay stage. The polarisation of each beam is controlled independently with a quarter wave plate (QWP) and a half wave plate (HWP). As indicated in Fig. $1 a$, two beamsplitters allow us to monitor the input and output of each beam. The light is coupled in and out of the SOA by two anti reflection-coated aspheric lenses mounted on micro-control 3D translation stages. The device under test is a commercially obtained $1.5 \mathrm{~mm}$ long bulk InGaAsP/InP travelling wave SOA biased at $350 \mathrm{~mA}$ and temperature regulated at $20^{\circ} \mathrm{C}$ by means of a Peltier cooler. An interference filter is used to isolate the probe signal from the broad ASE at $1580 \mathrm{~nm}$ and the signal is detected using a lock-in technique.

As most previous pump-probe studies of SOAs have been performed in a co-propagation configuration, it is useful to stress the main differences between co and counter propagation. While the transmission curves can look rather similar there are a few important differences. Unlike co-propagation where there is only one value of the delay for which the pulses overlap, in counter-propagation for a range of time delays, the pump and probe pulses overlap at different positions inside the SOA. This set-up is similar to the one previously presented for the study of polarisation dependent dynamics in [5], where more precision can be found.

This research has been supported by Enterprise Ireland under the Commercialisation Fund Technology Development programme, project number CFTD/06/IT/332. 
(a)

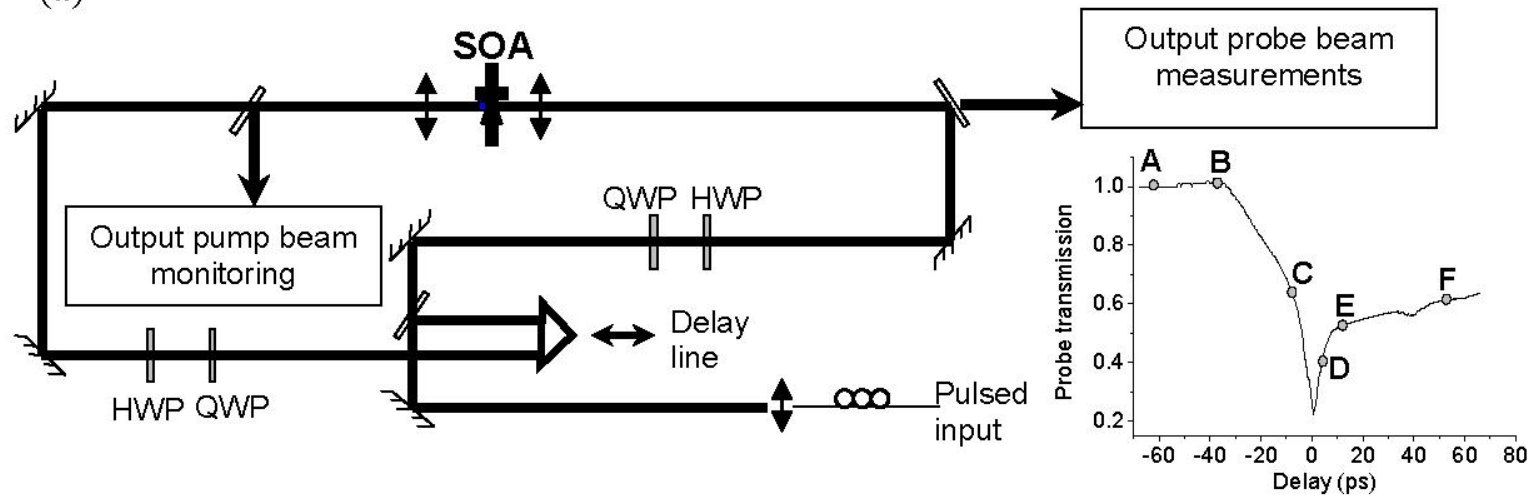

Fig. 1. (a) Experimental set-up. (b) A typically measurement of the probe transmission as a function of the time delay between the pump and probe pulses.

The pump and probe wavelength are set at $1580 \mathrm{~nm}$ at the peak of the SOA gain spectrum. The state of polarization is resolved at different delays. At a fixed pump-probe delay the transmitted probe intensity is recorded as a function of the orientation of the polarizer placed in front of the detector. The polarizer is rotated over $360^{\circ}$ in steps of $10^{\circ}$. A sample of the data, presented as a polar plot, is shown in figure 2 . The data is recorded at the six different values of pump-probe delay indicated on the probe transmission curve Fig. $1 b$ : $\mathbf{A}$, when the probe has exited the device before the pump enters, $\mathbf{B}$ at the onset of gain compression when pump and probe overlap at the output of the SOA, $\mathbf{C}$ where some gain compression has been achieved as pump and probe pulses overlap inside the device, $\mathbf{D}$ during the fast component of the gain recovery, $\mathbf{E}$ just after the fast gain recovery and $\mathbf{F}$ during the slow gain recovery component. No measurements were taken at minimum transmission due to presence of a coherent artefact. The data for delays $\mathrm{E}$ and $\mathrm{F}$ are found to be similar, showing little change in polarisation or amplitude. Therefore for clarity the measurements corresponding to $\mathrm{F}$ are not shown on the graphs.

\section{RESULTS AND DISCUSSION}

\subsection{Rotation of signals injected along an eigen axis}
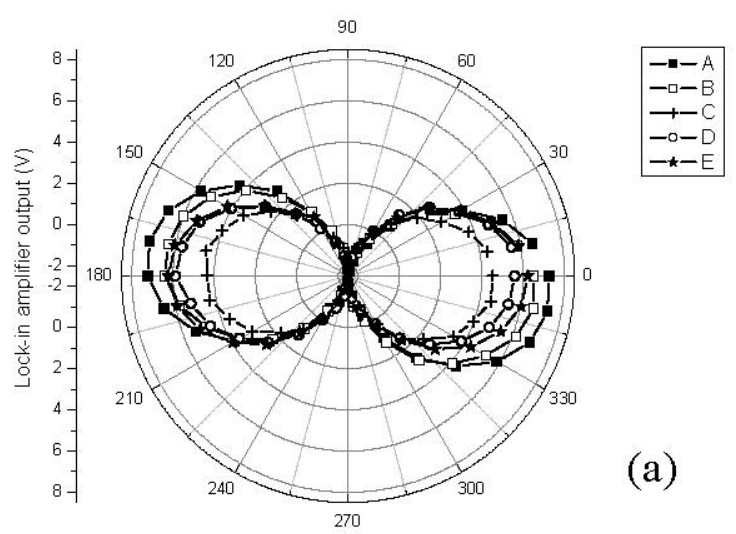

(a)
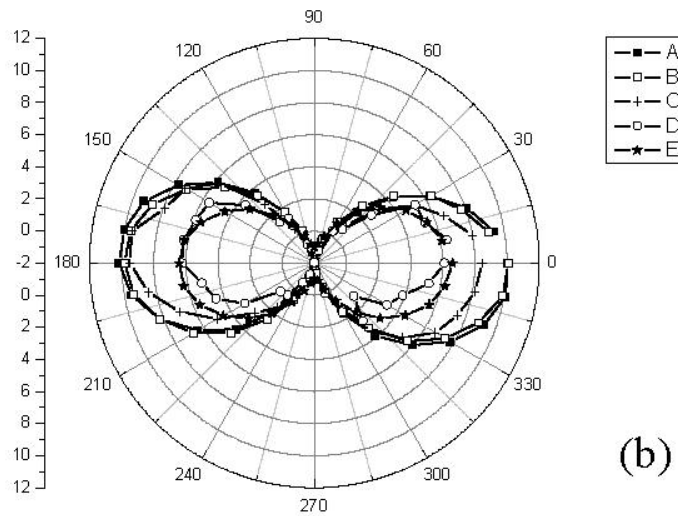

Fig. 2. Output intensity as a function of polarizer angle,

(a) probe TE, $12 \mathrm{fJ}$ and pump TE, $875 \mathrm{fJ}$, (b) probe TE, $12 \mathrm{fJ}$ and pump TM, $875 \mathrm{fJ}$.

To investigate whether or not there is any modification of the state of polarization for signals injected along an eigen mode both the pump and probe signals were injected along the eigen modes of the SOA. Results are shown in Fig. 2 for a $12 \mathrm{fJ}$ TE polarized probe and $875 \mathrm{fJ}$ pump (a) pump TE polarized, (b) pump TM polarized. The polar plots at six different delays are recorded and in this way any modification of the probe state of polarization can be determined. The state of polarization of the probe remains almost unchanged, linear and close to $0^{\circ}$ orientation, for all pumping conditions and pump-probe delays. At the highest pump power a small rotation of the probe state of polarization can be seen. It is always less than $10^{\circ}$ and too low to be used for polarisation switching. This small modification of the probe polarisation may be due to modes other than the fundamental 
propagating through the SOA [3]. The negative values on the polar plot corresponds to when the polarizer completely blocks the transmitted probe signal and a small residual amplified spontaneous emission signal modulated by the chopped probe signal is detected. The change in transmitted probe intensity at different pumpprobe delays corresponds to the probe gain compression and it is greater at high pump energies, as expected. While the level of gain compression achieved is strongly dependent on the pump and probe signal states of polarization (more details can be found in reference [5]), in all cases where the injected signal polarization corresponds to the device eigen mode the linear state of polarization is preserved after propagation through the device. It follows that if polarisation switching is to be used, the probe signal state of polarisation should be set away from the eigen modes of the device.

\subsection{Polarisation evolution}

For polarisation switching the combined effects of the polarisation dependent gain compression and the change of the probe state of polarisation have to be taken into account. In order to assess the potential for polarisation switching we examine the case where the pump and probe polarisations are set at $45^{\circ}$, with a probe pulse energy of $12 \mathrm{fJ}$ and for two different values of pump energy, $12 \mathrm{fJ}$ and $875 \mathrm{fJ}$, figures $3 a$ and $3 b$ respectively. As before the output probe intensity is then measured as a function of the output polarizer orientation at different pumpprobe delays and presented as polar plot. This allows us to monitor the change in output probe state of polarisation as a function of pump-probe delay.

As can be seen in Fig. $3 a$ for a low pump pulse energy, at delay position A the output probe signal is elliptical, then as the gain compression increases the signal becomes less elliptical and approximates a linear polarization. It remains in this state of polarization as the probe gain recovers with an almost constant orientation. In the high pump pulse energy case, Fig. $3 b$, again as is expected at delay position A the output probe has an elliptical state of polarization. As the gain compression increases it becomes almost circular at a delay of $-8 \mathrm{ps}$, delay position $\mathrm{C}$ and becomes linear as the device starts to recover and maintaining this polarization beyond $55 \mathrm{ps}$ (delay positions $\mathrm{D}$ and $\mathrm{E}$ ). The fact that the polarisation stays quasi linear as the device recovers indicates that the recovery of the probe state of polarization is dominated by the slower interband effects. Measurements performed with injected continuous wave laser beams, for which interband effects dominate, have also shown that the injection of a pump beam causes the output probe state of polarisation to become linear at this bias current. It is also interesting to note that although polarisation switching is often referred to in the literature as 'non-linear polarisation rotation', in this case it is mainly a change in the ellipticity of the signal that occurs and can be used for switching, while any rotation observed is small, typically less than $10^{\circ}$.
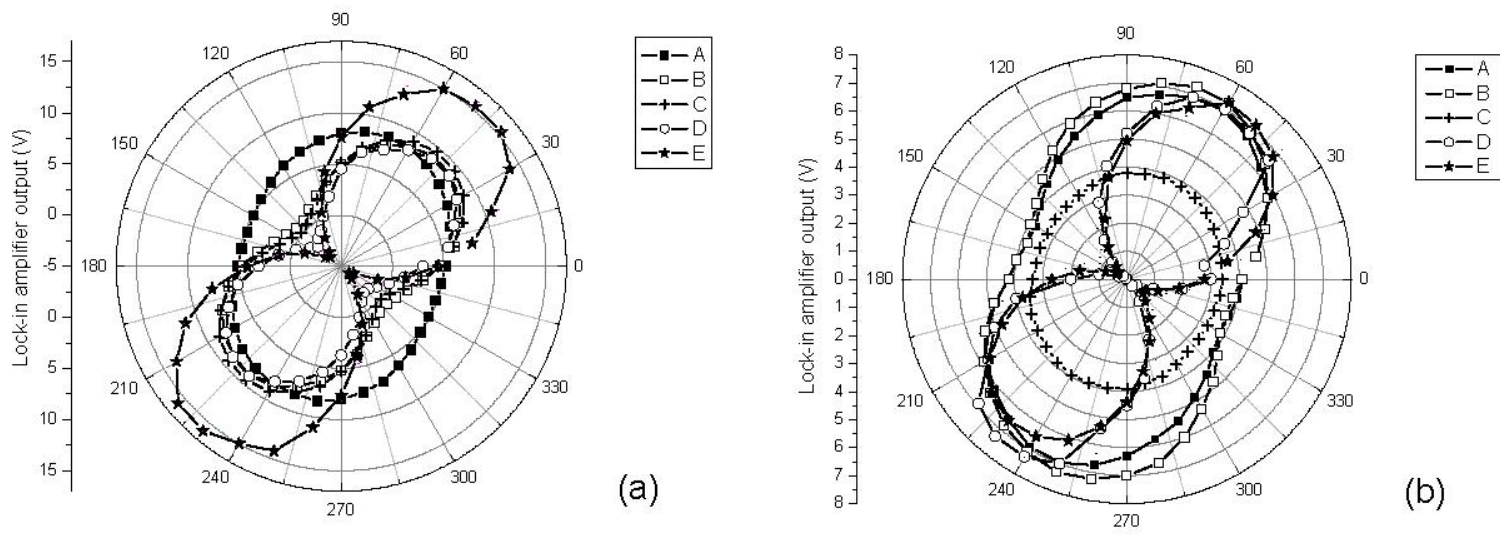

Fig. 3 Output intensity as a function of polarizer angle for probe and pump linearly polarised at $45^{\circ}$ (a) probe $12 \mathrm{fJ}$ and pump $12 \mathrm{fJ}$, (b) probe $12 \mathrm{fJ}$ and pump $875 \mathrm{fJ}$.

\subsection{Polarisation switching}

Examination of Fig. 3 clearly shows that very different polarization switching performances can be obtained depending on the orientation of the output polarizer. For example in the high power case, Fig. $3 b$, at $0^{\circ}$ the change in probe transmission as a function of pump-probe delay seems quite small while the extinction ratio at $320^{\circ}$ would be very high (limited only by the extinction ratio of the polarizer), since the probe output is linear with a $50^{\circ}$ orientation at pump-probe delay position D. However, under this condition the recovery of the intensity is dominated by the slow gain recovery dynamics. A polarizer selecting the probe signal at an orientation of $45^{\circ}$ would results in a faster recovery but at the cost of a lower extinction co-efficient. Examination of the polarisation resolved probe transmission decays at this orientation allows the switching performance to be assessed. As shown on Fig. $4 a$, placing a polarizer at $0^{\circ}$ deteriorates the performance by increasing the minimum pump transmission from 0.2 to 0.5 , while placing a polarizer at $45^{\circ}$ improves the performance by decreasing the transmission from 0.2 to 0.01 and by dramatically speeding up the recovery. In 
fact the intensity recovers to its initial value in approximately $5 \mathrm{ps}$ in this case. It is important to remember here that recovery of the intensity does not imply recovery of the device, as is clearly evidenced by the polar plots in figure 3. It is actually the result of the change in polarisation experienced by the probe signal compensating for the slow gain recovery. The trends observed at higher pump power Fig. $4 b$ are the same. Yet again the introduction of a polarizer at $45^{\circ}$ increases the switching extinction ratio achievable and results in a faster recovery, though clearly the benefits are less than with a low pump power.
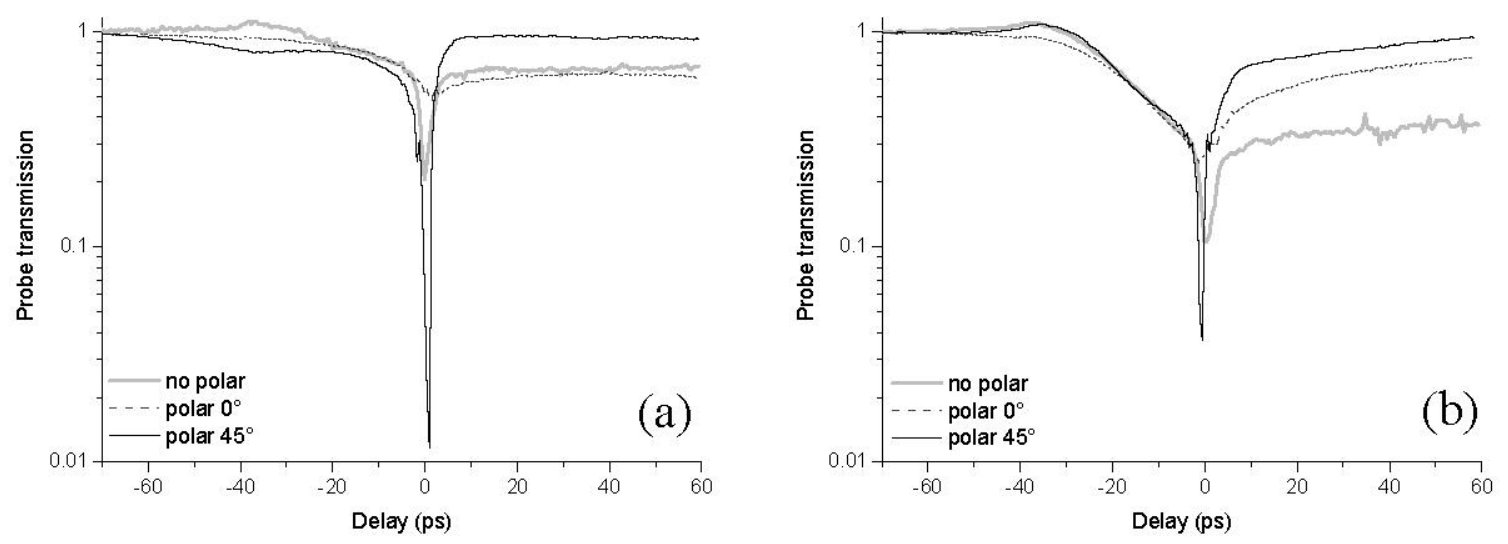

Fig. 4. Polarisation resolved decays, pump and probe linearly polarized at $45^{\circ}$, (a) probe $12 \mathrm{fJ}$ and pump $12 \mathrm{fJ}$, (b) probe $12 \mathrm{fJ}$ and pump $875 \mathrm{fJ}$.

\section{CONCLUSION}

In this paper we have presented a study of the dynamics of non-linear polarisation rotation using a free space contra-propagation configuration. For signals injected along the eigen modes of the device any rotation of the state of polarization is less than $10^{\circ}$, and found to be too small for polarisation switching. The signals' polarisations were set to $45^{\circ}$ and changes in probe state of polarisation were observed as a function of the pumpprobe delay. It was demonstrated that even though the recovery of the polarisation is dominated by the slower interband effects a fast recovery of the probe transmission level can still be achieved by careful selection of the output polarizer orientation. The most promising switching configuration can be determined using a combined study of polar plots and polarisation resolved decays. In conclusion polarisation switching can be used to enhance the performance of gain switching schemes, both in terms of extinction ratio and speed.

\section{REFERENCE}

[1] Durhuus, B. Mikkelsen, C. Joergensen, S.L. Danielsen, and K.E. Stubkjaer, "All-optical wavelength conversion by semiconductor optical amplifiers," Joumal of Lightwave Technology, vol. 14, pp. 942-954, 1996.

[2] D. Lenstra, Y. Liu, M. T. Hill, G.-D. Khoe, and H.J.S. Dorren, "Nonlinear polarization rotation in semiconductor optical amplifiers: Theory and application to all-optical flip-flop memories, "IEEE Joumal of Quantum Electronics, vol. 39, pp. 141-148, 2003.

[3] H. Soto, J.C. Dominguez, Erasme D., and G. Guekos, "Demonstration of an all-optical switch using crosspolarization modulation in semiconductor optical amplifiers," Microwave and Optics Technology Letters, vol. 29, (3), pp. 205-209, 2001.

[4] M.F.C. Stephens, M. Asghari, R.V. Penty, and I.H. White, "Demonstration of ultrafast all-optical wavelength conversion utilizing birefringence in semiconductor optical amplifiers," IEEE Photonics Technology Letters, vol. 9, pp. 449-451, 1997.

[5] S. Philippe, A.L. Bradley, F. Surre, P. Landais, R. Maldonado-Basilio, H. Soto-Ortiz, B.F. Kennedy, "Time resolved polarization dependence of intra-band effects in semiconductor optical amplifier", submitted to IEEE J. Quant. Electron., April 2007. 